\title{
Guía de práctica clínica para el tamizaje y manejo del episodio depresivo leve en el primer nivel de atención del Seguro Social del Perú (EsSalud)
}

\author{
Clinical practice guideline for the screening and \\ management of the mild depressive episode at the \\ first level of care for the Peruvian Social Security \\ (EsSalud)
}

Correspondencia Héctor Garavito-Farro hector.garavito@essalud.gob.pe

Recibido: 24/08/2020

Arbitrado por pares

Aprobado: 24/09/2020

Citar como: Macciota-Felices B, Morón-Corales C, Luna-Matos M, Gonzales-Madrid V, MelgarejoMoreno A, Zafra-Tanaka JH, et al. Guía de práctica clínica para el tamizaje y manejo del episodio depresivo leve en el primer nivel de atención del Seguro Social del Perú (EsSalud). Acta Med Peru. 2020;37(4):536-47. doi: doi: https://doi.org/10.35663/ amp.2020.374.1648
Beatrice Macciotta-Felices ${ }^{1,2}$, Carla Morón-Corales ${ }^{3}$, Matilde Luna-Matos ${ }^{4}$, Víctor Gonzales-Madrid ${ }^{5}$, Amelia Melgarejo-Moreno ${ }^{6}$, Jessica H. ZafraTanaka², Sergio Goicochea-Lugo², Raisa N. Martinez-Rivera', Wendy NietoGutierrez², Fabián Fiestas-Saldarriaga², Alvaro Taype-Rondan², Raúl TimanáRuiz², Hector Garavito-Farro²

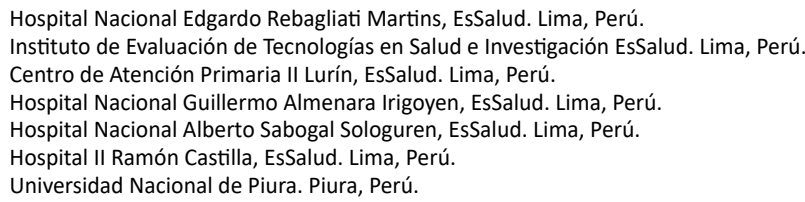

\section{RESUMEN}

El presente artículo resume la guía de práctica clínica (GPC) para el tamizaje y el manejo del episodio depresivo leve en el primer nivel de atención en el Seguro Social del Perú (EsSalud). Para el desarrollo de esta GPC, se conformó un grupo elaborador de la guía (GEG) que incluyó especialistas clínicos y metodólogos, el cual formuló 06 preguntas clínicas. Para responder cada pregunta se realizó búsquedas sistemáticas en PubMed y en repositorios de GPC, y se seleccionó la evidencia pertinente. La certeza de la evidencia fue evaluada usando la metodología Grading of Recommendations Assessment, Development, and Evaluation (GRADE). En reuniones periódicas, el GEG usó la metodología GRADE para revisar la evidencia y emitir las recomendaciones. Se emitieron siete recomendaciones (tres fuertes y cuatro condicionales), 28 puntos de buena práctica clínica, y dos flujogramas.

Palabras claves: Síntomas depresivos; Guía de práctica clínica; Enfoque GRADE; Medicina basada en la evidencia (fuente: DeCS BIREME). 


\section{ABSTRACT}

This paper summarizes the clinical practice guide (CPG) for the screening and management of mild depressive episode at the first level of care in the Social Security of Peru (EsSalud). A guideline development group (GDG) was established for develop this CPG, which included clinical and methodology specialists, who formulated 06 clinical questions. Systematic searches were conducted in Pubmed and GPC repositories to answer each question, and relevant evidence was selected. The certainty of the evidence was assessed using the Grading of Recommendations Assessment, Development, and Evaluation (GRADE) methodology. In periodic work meetings, the GDG used the GRADE methodology for reviewing the evidence and for developing recommendations. At the end, this CPG formulated 07 recommendations ( 03 strong and 04 conditional),

28 points of good clinical practice, and 02 flow charts were formulated.

Keywords: Depressive episode; Practice guideline; GRADE approach; Evidence-based medicine (source: MeSH NLM).

\section{INTRODUCCIÓN}

La depresión se define siguiendo los criterios de sistemas internacionales, siendo los más conocidos el sistema de Clasificación Internacional de Enfermedades en su décima edición (CIE-10) y la quinta edición del Manual Diagnóstico y Estadístico de los Trastornos Mentales (DSM-5). Si bien ambos sistemas tienen criterios y clasificación de la severidad similares, el término usado para referirse a la depresión varía entre ellas. Mientras que la CIE-10 define a este trastorno como "episodio depresivo" [1], el DSM-5 lo define como "depresión mayor" [2].

La depresión afecta a más de 300 millones de personas en el mundo, siendo considerada como la principal causa mundial de discapacidad ${ }^{[3]}$. El episodio depresivo leve es un subtipo de trastorno depresivo que suele omitido, pues entre el 50-70\% de estos pacientes no son diagnosticados ${ }^{[4]}$. Sin embargo, puede generar deficiencias en la funcionalidad del individuo $y$, consecuentemente, una disminución en su calidad de vida ${ }^{[5]}$.

En Perú, la prevalencia ajustada por edad de tener depresión en el 2018, evaluada con el instrumento Patient Health Questionnaire-9 (PHQ-9), fue de 6,2\% ${ }^{[6]}$. Desde el 2015 se vienen implementando políticas centradas en el primer nivel de atención para la mejora de la salud mental ${ }^{[7]}$. Sin embargo, no se han evidenciado cambios en la tendencia de episodios depresivos del 2014 al $2018^{[6]}$.

Debido a la importancia de la depresión a nivel mundial y en el contexto peruano, la Dirección de Guías de Práctica Clínica, Farmacovigilancia y Tecnovigilancia del Instituto de Evaluación de Tecnologías en Salud e Investigación (IETSI) del Seguro Social del Perú (EsSalud), elaboró una guía de práctica clínica (GPC) basada en evidencias para el tamizaje y el manejo del episodio depresivo leve en el primer nivel de atención, cuyas recomendaciones deben ser aplicadas por profesionales de la salud en EsSalud (Tabla 1). El presente artículo es un resumen de dicha GPC.

\section{METODOLOGÍA}

El procedimiento seguido para la elaboración de la presente GPC está detallado en su versión "in extenso", la cual puede descargarse de la página web del IETSI de EsSalud (http://www. essalud.gob.pe/ietsi/guias_pract_clini.html).

En resumen, se aplicó la siguiente metodología:

Conformación del grupo elaborador de la guía local (GEG): Se conformó un GEG, que incluyó metodólogos, médicos psiquiatras, médicos de familia y psicólogos.

Formulación de preguntas: En concordancia con los objetivos y alcances de esta GPC, el GEG formuló 6 preguntas clínicas (Tabla 2), cada una de las cuales pudo tener una o más preguntas PICO (Population, Intervention, Comparator, Outcome). A su vez, cada pregunta PICO pudo tener uno o más desenlaces (o outcomes) de interés.

Búsqueda y selección de la evidencia: Para cada pregunta PICO, se realizó la búsqueda y selección de la evidencia. Para ello, durante el 2019 se buscó revisiones sistemáticas (RS) publicadas como artículos científicos (mediante búsquedas sistemáticas en PubMed) o realizadas como parte de una GPC previa (mediante una búsqueda sistemática de GPC sobre el tema) (Material suplementario 1). Cuando se encontraron RS de calidad aceptable, se escogió una RS para la toma de decisiones, la cual fue actualizada cuando el GEG lo consideró necesario. Cuando no se encontró ninguna RS de calidad aceptable, se realizó una búsqueda de novo de estudios primarios.

Evaluación de la certeza de la evidencia: La certeza de la evidencia para cada desenlace de cada pregunta PICO pudo ser alta, moderada, baja, o muy baja (Tabla 2). Para evaluar la certeza de la evidencia, se siguió la metodología de Grading of Recommendations Assessment, Development, and Evaluation (GRADE) ${ }^{[8]}$. Para presentar el resumen de los estudios, se usó tablas de resumen de evidencias (Summary of Findings) de GRADE (Material suplementario 2). 
Tabla 1. Lista de recomendaciones y puntos de buena práctica clínica.

Número*

Enunciado

Pregunta 1: En población adulta, ¿se deberían brindar intervenciones para prevenir el desarrollo de episodios depresivos?

Rec-1.1

En población adulta del primer nivel de atención, sugerimos brindar intervenciones para prevenir el desarrollo de episodios depresivos.

BPC-1.1

Brindar intervenciones preventivas de tipo psicológica y educativa basadas en terapia cognitivo conductual como, por ejemplo: charlas sobre desarrollo de la inteligencia emocional, estrategias que faciliten la resolución de problemas personales, actitudes que debe adoptar la persona para afrontar dichos problemas, entre otros.

BPC-1.2

Brindar intervenciones preventivas de tipo psicológica y educativa principalmente a grupos de riesgo: adultos mayores, pacientes con enfermedades crónicas, mujeres en periodo de periparto, y a aquellos pacientes que tengan síntomas depresivos pero que no cumplan los criterios para episodio depresivo según CIE-10.

Pregunta 2: En población adulta, ¿se debería realizar tamizaje de episodio depresivo durante la consulta en el primer nivel de atención?

Rec-2.1

En grupos prioritarios que acuden a consulta en el primer nivel de atención, recomendamos realizar tamizaje de episodio depresivo.

BPC-2.1

Los grupos prioritarios a considerar son: adultos mayores, pacientes con enfermedades crónicas, y mujeres en periodo de periparto.

BPC-2.2

En grupos prioritarios que acuden a consulta en el primer nivel de atención, utilizar el instrumento PHQ-9 para realizar el tamizaje de episodio depresivo. Considerar como positivo cuando se obtenga un puntaje mayor o igual a 10.

BPC-2.3

En grupos prioritarios que acuden a consulta en el primer nivel de atención, cualquier personal de salud que tenga la oportunidad de atender al paciente podrá realizar el tamizaje de episodio depresivo.

BPC-2.4

En grupos prioritarios que acuden a consulta en el primer nivel de atención con tamizaje positivo, confirmar el episodio depresivo con los criterios $\mathrm{CIE}-10$ según la normativa nacional vigente (ver tabla en el desarrollo de la pregunta 2 de versión extensa).

En pacientes adultos con episodio depresivo confirmado, realizar el descarte de depresión bipolar. Considerar depresión bipolarsise sospecha de cualquiera de las siguientes situaciones:

Ocurrencia de un episodio hipomaníaco (elevación leve y persistente del ánimo) pasado o presente cuyos síntomas estuvieron presentes por cuatro a más días.

Ocurrencia de un episodio maníaco (humor elevado sin relación a las circunstancias, que puede variar a una excitación casi incontrolable) cuyos síntomas estuvieron presentes por una o más semanas.

BPC-2.6

En pacientes adultos con episodio depresivo confirmado en quienes se sospeche de depresión bipolar, referir al psiquiatra para diagnóstico y manejo.

En pacientes adultos con episodio depresivo no bipolar, se definirá la severidad del episodio depresivo con el instrumento PHQ-9:

BPC-2.7 Puntaje de 10-14: episodio depresivo leve

Puntaje de 15-19: episodio depresivo moderado

Puntaje de 20-27: episodio depresivo severo

BPC-2.8 En pacientes adultos con episodio depresivo moderado o severo, referir al psiquiatra para manejo.

Pregunta 3: En pacientes adultos con episodio depresivo leve, ¿qué terapia se debería brindar como manejo inicial: terapia farmacológica o psicoterapia?

Rec-3.1

En pacientes adultos con episodio depresivo leve, sugerimos brindar psicoterapia y/o farmacoterapia como manejo inicial teniendo en cuenta la existencia de alguna contraindicación para el uso de alguna de estas terapias, las preferencias del paciente y la disponibilidad de recursos humanos y logísticos. 
Tabla 1. Lista de recomendaciones y puntos de buena práctica clínica. (viene de la pág. 538)

Pregunta 4: En pacientes adultos con episodio depresivo leve en los que se opte por iniciar el manejo con psicoterapia, ¿qué psicoterapia se debería usar?

Rec-4.1

En pacientes adultos con episodio depresivo leve en los que se opte por iniciar el manejo con psicoterapia, recomendamos prescribir terapia cognitivo conductual.

Rec-4.2 En pacientes adultos con episodio depresivo leve en los que se opte por iniciar el manejo con psicoterapia sugerimos prescribir terapia de resolución de problemas cuando no esté disponible la terapia cognitivo conductual. En pacientes adultos con episodio depresivo leve en los que se opte por iniciar el manejo con psicoterapia,

BPC-4.1 considerar el uso de otras psicoterapias (activación conductual, terapia psicodinámica o terapia interpersonal), solo como un complemento de las terapias cognitivo conductual o terapia de resolución de problemas.

BPC-4.2 En pacientes adultos con episodio depresivo leve en quienes se sospeche un entorno familiar disfuncional, considerar el uso de la terapia familiar para evitar el reforzamiento de los síntomas depresivos.

BPC-4.3 En pacientes adultos con episodio depresivo leve en los que se opte por iniciar el manejo con psicoterapia, considerar la terapia grupal para fortalecer la red de apoyo de los pacientes.

En pacientes adultos con episodio depresivo leve en los que se opte por iniciar el manejo con psicoterapia, la

BPC-4.4 duración de la terapia será de 8 hasta 20 sesiones (con una frecuencia de una a dos veces por semana), y ampliar dicho periodo dependerá de las necesidades del paciente y del profesional de salud que la realice.

En pacientes adultos con episodio depresivo leve en los que se opte por iniciar el manejo con psicoterapia, medir el puntaje de severidad basal de los síntomas con el instrumento PHQ-9 antes de iniciar la psicoterapia. Posteriormente, evaluar la respuesta a la terapia midiendo el cambio en el puntaje del PHQ-9, al menos en la sexta sesión de psicoterapia:

BPC-4.5 - Respuesta a la terapia: disminución de 50\% a más en el puntaje basal de severidad de los síntomas.

- Respuesta parcial a la terapia: disminución entre el 20 a $49 \%$ en el puntaje basal de severidad de los síntomas.

- No respuesta a la terapia: disminución de $19 \%$ o menos en el puntaje basal de severidad de los síntomas. hayan alcanzado respuesta a la terapia a partir de la sexta sesión, continuar el manejo hasta completar el número de sesiones planificado. De lo contrario, reformular el plan psicoterapéutico.

En pacientes adultos con episodio depresivo leve en los que se opte por iniciar el manejo con psicoterapia,

BPC-4.7 reevaluar la respuesta a la terapia entre la sesión 8 a 20. De haber alcanzado respuesta, evaluar remisión durante las tres semanas posteriores al término de la psicoterapia. De lo contrario, evaluar la presencia de una patología subyacente y la progresión de la severidad del trastorno depresivo (episodio depresivo moderado o severo).

En pacientes adultos con episodio depresivo leve, se definirá remisión en la siguiente situación:

BPC-4.8 Ausencia de los síntomas característicos de depresión durante al menos tres semanas.

Pudiendo estar presentes hasta tres de los síntomas depresivos restantes (ver tabla en el desarrollo de la pregunta 2 de versión extensa).

En pacientes adultos con episodio depresivo leve en los que se opte por iniciar el manejo con psicoterapia que

BPC-4.9 hayan alcanzado remisión de los síntomas, dar de alta. De lo contrario, evaluar la presencia de una patología subyacente y la progresión de la severidad del trastorno depresivo (episodio depresivo moderado o severo).

Pregunta 5: En pacientes adultos con episodio depresivo leve en los que se opte por iniciar el manejo con terapia farmacológica, ¿con qué antidepresivo se debería iniciar la terapia?

Rec-5.1

En pacientes con episodio depresivo leve en los que se opte por iniciar el manejo con terapia farmacológica, sugerimos iniciar con un inhibidor selectivo de la recaptación de serotonina (ISRS).

BPC-5.1

En pacientes con episodio depresivo leve en los que se opte por iniciar el manejo con terapia farmacológica, no iniciar el tratamiento con clomipramina o amitriptilina dada su baja aceptabilidad y por requerir evaluación cardiológica previa a su uso dado el riesgo de complicaciones como infarto agudo de miocardio y arritmias. 
Tabla 1. Lista de recomendaciones y puntos de buena práctica clínica. (viene de la pág. 539)

Número* Enunciado

En pacientes con episodio depresivo leve que no toleren los efectos adversos de la terapia con uno de los ISRS prescribir otro ISRS u otro grupo de antidepresivos teniendo en cuenta las comorbilidades, preferencias del paciente, interacciones con otros fármacos y el perfil de adherencia al antidepresivo.

En pacientes adultos con episodio depresivo leve en los que se opte por iniciar el manejo con terapia farmacológica,

BPC-5.3 la duración de la terapia será de 8 hasta 12 semanas y ampliar dicho periodo dependerá de las necesidades del paciente y del profesional de salud que la realice.

En pacientes adultos con episodio depresivo leve en los que se opte por iniciar el manejo con terapia farmacológica,

BPC-5.4 medir el puntaje de severidad basal de los síntomas depresivos con el instrumento PHQ- 9 antes de iniciar la terapia farmacológica. Posteriormente, evaluar la respuesta a la terapia al menos entre la cuarta a sexta semana de iniciado el manejo, usando el instrumento PHQ-9 (definición en BPC-4.5)

En pacientes adultos con episodio depresivo leve en los que se opte por iniciar el manejo con terapia farmacológica que hayan alcanzado respuesta a la terapia entre la cuarta y sexta semana de tratamiento, mantener la dosis del antidepresivo y continuar la terapia. De lo contrario, incrementar la dosis del antidepresivo y continuar la terapia.

En pacientes adultos con episodio depresivo leve en los que se opte por iniciar el manejo con terapia farmacológica, reevaluar la respuesta a la terapia entre la octava y decimosegunda semana de iniciado el manejo. Si alcanzaron

BPC-5.6 respuesta a la terapia entre la octava a decimosegunda semana de tratamiento, continuar brindando el antidepresivo a la misma dosis durante las siguientes tres semanas en las que se evalúa la remisión (definición en BPC 18). De lo contrario, evaluar la presencia de una patología subyacente y la progresión de la severidad del trastorno depresivo (episodio depresivo moderado o severo).

En pacientes adultos con episodio depresivo leve en los que se opte por iniciar el manejo con terapia farmacológica que hayan alcanzado remisión de los síntomas, considerar continuar la farmacoterapia durante al menos seis

BPC-5.7 meses, luego de lo cual reducir gradualmente la dosis del antidepresivo hasta el cese. De lo contrario, evaluar la presencia de una patología subyacente y la progresión de la severidad del trastorno depresivo (episodio depresivo moderado o severo).

Pregunta 6: En pacientes adultos con episodio depresivo leve, ¿se debería indicar realizar ejercicio físico?

Rec-6.1 En pacientes adultos con episodio depresivo leve, recomendamos indicar la realización de ejercicio físico.

BPC-6.1 En pacientes con episodio depresivo leve, prescribir ejercicios como caminar, trotar o correr durante 30 minutos, tres veces por semana, de acuerdo con la condición física de cada paciente.

BPC-6.2 En pacientes con episodio depresivo leve, la actividad física se prescribirá como un complemento de la terapia farmacológica o psicoterapia.

* Número de recomendación (R) y de punto de buena práctica clínica (BPC)

** Punto de buena práctica clínica (BPC)

*** La certeza de la evidencia solo se establece para las recomendaciones, mas no para los puntos de BPC

Formulación de las recomendaciones: El GEG revisó la evidencia seleccionada para cada pregunta clínica en reuniones periódicas, y formuló recomendaciones fuertes o condicionales usando la metodología GRADE (Tabla 2) ${ }^{[9]}$. Para ello, se tuvo en consideración: 1) Beneficios y daños de las opciones, 2) Valores y preferencias de los pacientes, 3) Aceptabilidad por parte de los profesionales de salud, 4) Factibilidad de las opciones en los establecimientos de salud de EsSalud, y 5) Uso de recursos. Luego de discutir estos criterios para cada pregunta, el GEG formuló las recomendaciones por consenso o por mayoría simple. Asimismo, el GEG formuló puntos de buena práctica clínica (BPC), usualmente en base a su experiencia clínica. Se le asignó a cada recomendación el nivel de certeza más bajo alcanzado por alguno de los desenlaces críticos de su pregunta.
Revisión por expertos externos: La presente GPC fue revisada en reuniones con médicos especialistas representantes de otras instituciones y tomadores de decisiones. Asimismo, su versión in-extenso fue enviada por vía electrónica a expertos externos para su revisión (mencionados en la sección de agradecimientos). El GEG tuvo en cuenta los resultados de estas revisiones para modificar las recomendaciones finales.

Aprobación de la GPC: La presente GPC fue aprobada para su uso en EsSalud, con Resolución N¹12-IETSI-ESSALUD-2019.

Actualización de la GPC: La presente GPC tiene una vigencia de tres años. Al acercarse al fin de este período, se procederá a realizar una RS de la literatura para su actualización, luego de la cual se decidirá si se actualiza la presente GPC o se procede a realizar una nueva versión. 
Tabla 2. Significado de los niveles de certeza de la evidencia y de la fuerza de la recomendación.

Categorías Significado

\begin{tabular}{|c|c|}
\hline \multicolumn{2}{|l|}{ Certeza de la evidencia } \\
\hline $\begin{array}{l}(\bigoplus \oplus \oplus \oplus) \\
\quad \text { Alta }\end{array}$ & Es muy probable que el verdadero efecto sea similar al efecto estimado. \\
\hline $\begin{array}{l}(\oplus \oplus \oplus \bigcirc) \\
\text { Moderada }\end{array}$ & $\begin{array}{l}\text { Es moderadamente probable que el verdadero efecto sea similar al efecto estimado, pero es } \\
\text { posible que sea sustancialmente diferente }\end{array}$ \\
\hline $\begin{array}{l}(\bigoplus \oplus \bigcirc) \\
\text { Ваја }\end{array}$ & $\begin{array}{l}\text { Nuestra confianza en el efecto es limitada. El verdadero efecto podría ser sustancialmente } \\
\text { diferente al efecto estimado }\end{array}$ \\
\hline $\begin{array}{l}(\bigoplus \bigcirc \bigcirc \bigcirc) \\
\text { Muy baja }\end{array}$ & $\begin{array}{l}\text { Nuestra confianza en el efecto es pequeña. El verdadero efecto probablemente sea sustancialmente } \\
\text { diferente al efecto estimado }\end{array}$ \\
\hline \multicolumn{2}{|l|}{ Fuerza de la recomendación } \\
\hline Recomendación fuerte & $\begin{array}{l}\text { El GEG considera que todos o casi todos los profesionales que revisan la evidencia disponible } \\
\text { seguirían esta recomendación. En la formulación de la recomendación se usa el término "se } \\
\text { recomienda" }\end{array}$ \\
\hline Recomendación Condicional & $\begin{array}{l}\text { El GEG considera que la mayoría de los profesionales que revisan la evidencia disponible seguirían } \\
\text { esta recomendación, pero un grupo de profesionales podría optar por no aplicarlas en alguna } \\
\text { población particular, siempre que sea justificado. En la formulación de la recomendación se usa } \\
\text { el término "se sugiere" }\end{array}$ \\
\hline
\end{tabular}

\section{RECOMENDACIONES}

La presente GPC abordó 6 preguntas clínicas, con relación a cuatro temas: tamizaje, diagnóstico, manejo y prevención. En base a dichas preguntas se formularon 7 recomendaciones (3 fuertes y 4 condicionales), 28 BPC, y 2 flujogramas (uno de tamizaje y diagnóstico, y otro de manejo) (Tabla 1, Figura 1 y 2).

La presente GPC se enfoca en el tamizaje, diagnóstico, manejo y prevención de los pacientes que llegan al primer nivel de atención, expuestos a desarrollar o tener episodio depresivo leve. Para este problema de salud, se busca brindar intervenciones de prevención (pregunta 1) y tamizaje (pregunta 2) de episodio depresivo leve; y, en los casos diagnosticados, definir si realizar psicoterapia o terapia farmacológica (pregunta 3), qué psicoterapia usar (pregunta 4), qué terapia farmacológica usar (pregunta 5), y la utilidad del ejercicio físico (pregunta 6).

A continuación, se expondrá las recomendaciones para cada pregunta clínica, así como un resumen del razonamiento seguido para llegar a cada recomendación. Todos los enunciados (recomendaciones o BPC) se encuentran enumerados en la Tabla 1, por lo cual en el texto solo se mencionará el número del enunciado al cual se hace referencia. Los datos numéricos de los beneficios y daños para cada pregunta se exponen en las tablas SoF, que se pueden consultar en el Material suplementario 2.

Pregunta 1: En población adulta, ¿̇se deberían brindar intervenciones para prevenir el desarrollo de episodios depresivos?

\section{Rec-1.1:}

Para contestar esta pregunta se consideró la RS de Conejo-Cerón $2017^{[10]}$ en la que se incluyeron 13 ensayos clínicos aleatorizados (ECA) con una población de adultos mayores de 18 años sin depresión. Se evaluaron los efectos de diversas intervenciones psicológicas (basadas en terapia cognitivo conductual y terapia interpersonal) y/o educativas (charlas y lecturas) comparadas con el cuidado usual (tratamiento farmacológico y tratamiento psicológico) o placebo.

Si bien se consideró que el beneficio de estas intervenciones fue pequeño, el GEG tomó en cuenta que los grupos de riesgo (adultos mayores, pacientes con enfermedades crónicas, mujeres en período de periparto, y pacientes con síntomas depresivos que no cumplan con todos los criterios de episodio depresivo) podrían obtener beneficios considerables mayores comparados con los posibles daños. Por lo cual, se decidió emitir una recomendación a favor de brindar intervenciones psicológicas y educativas para prevenir el desarrollo de episodios depresivos. Sin embargo, debido a que la certeza de la evidencia fue baja y a que su implementación requerirá inversión de recursos y tiempo para capacitación al personal de salud, se asignó una fuerza condicional a la recomendación.

BPC-1.1: Las intervenciones preventivas que se implementen deben estar basadas en terapia cognitivo conductual (TCC) ya que fueron las principalmente evaluadas ${ }^{[10,11]}$.

BPC-1.2: Brindar intervenciones preventivas principalmente a los grupos de riesgo como adultos mayores, pacientes con enfermedades crónicas, mujeres en periodo de periparto, y a aquellos pacientes que tengan síntomas depresivos pero que no cumplan los criterios para episodio depresivo según el CIE-10 ${ }^{[12]}$. 


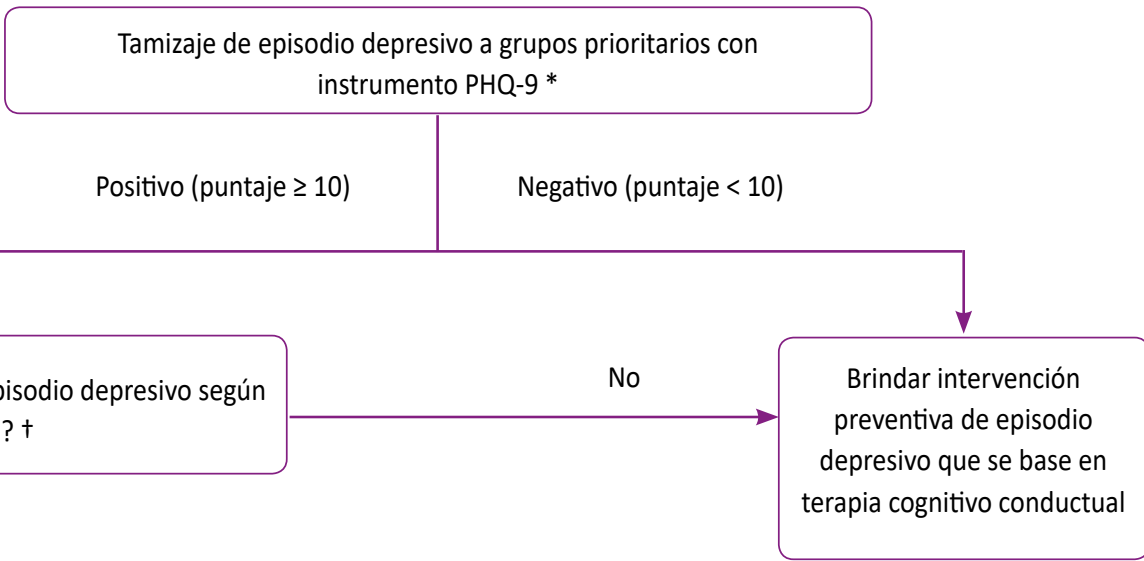

¿Se sospecha de depresión bipolar?

- Ocurrencia de un episodio hipomaníaco (elevación leve y persistente del ánimo) pasado o presente cuyos síntomas estuvieron presentes por cuatro a más días

$\mathrm{u}$

- Ocurrencia de un episodio maníaco (humor elevado sin relación a las circunstancias, que puede variar a una excitación casi incontrolable) cuyos síntomas estuvieron presentes por una o más semanas

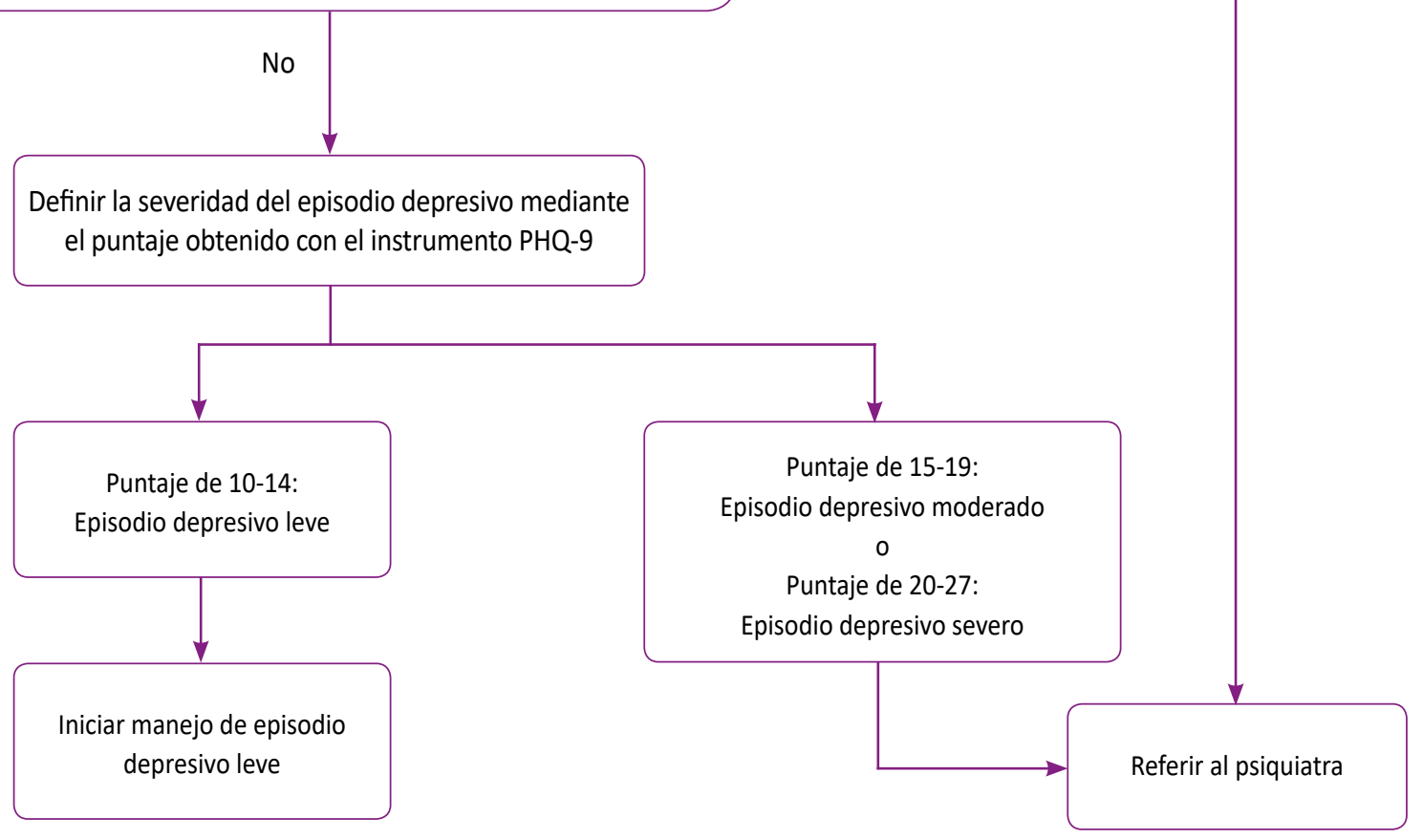

CIE-10: Clasificación Internacional de Enfermedades, 10ma edición; PHQ-9: Patient Health Questionnaire-9.

* Los grupos prioritarios a considerar son: adultos mayores, pacientes con enfermedades crónicas, y mujeres en periodo de periparto

+ La tabla de criterios diagnósticos de episodio depresivo según CIE-10 se encuentra en el desarrollo de la pregunta 2.

Figura 1. Flujograma de tamizaje y diagnóstico del episodio depresivo leve. 
Pregunta 2: En población adulta, ¿se debería realizar tamizaje de episodio depresivo durante la consulta en primer nivel de atención?

\section{Rec-2.1:}

Para contestar esta pregunta se tomó en consideración el ECA de Williams $1999^{[13]}$ para los desenlaces de número de síntomas depresivos y recuperación, y el ECA de Silverstone $2017^{[14]}$ para el desenlace de síntomas depresivos.

El ECA de Silverstone 2017 evaluó el efecto de una estrategia de tamizaje en la disminución de síntomas depresivos evaluados con el instrumento PHQ-9, y para ello comparó cuatro brazos: 1) control, definido como tamizaje, pero sin entrega de los resultados del mismo; 2) tamizaje más cuidado habitual; 3) tamizaje más terapia cognitivo conductual virtual; y 4) tamizaje más cuidado escalonado. Al comparar los puntajes de PHQ-9 a las 12 semanas de seguimiento entre el grupo que recibió cuidado habitual y el grupo que recibió tamizaje con PHQ-9, no se halló diferencias estadísticamente significativas.

El ECA de Williams 1999 evaluó el efecto de un programa de tamizaje en el número de síntomas depresivos de acuerdo a los criterios de Diagnostic and Statistical Manual of Mental Disorders-III (DSM III), y en la recuperación, definida como la disminución síntomas del DSM III. Sin embargo, no se encontró diferencias estadísticamente significativas ${ }^{[13]}$.

Si bien estos estudios no encontraron beneficio en el realizar tamizaje, el GEG consideró que los estudios evaluados fueron realizados en muestras pequeñas, hubo pérdida de participantes durante el seguimiento, y no se define claramente qué cuidados recibió el grupo control "cuidado usual". Sin embargo, realizar tamizaje permitiría un diagnóstico oportuno y la disminución de complicaciones del episodio depresivo ${ }^{[12]}$. Por este motivo el GEG optó por emitir una recomendación a favor de realizar el tamizaje. Por otro lado, la implementación del tamizaje es factible, y podría generar ahorros a largo plazo al evitar posibles hospitalizaciones y complicaciones futuras en los pacientes. Por este motivo, a pesar de la certeza de la evidencia muy baja, se decidió emitir una recomendación fuerte.

BPC-2.1: El beneficio de una estrategia de tamizaje sería mayor en grupos de riesgo prioritarios para presentar depresión como los adultos mayores, pacientes con enfermedades crónicas, y mujeres en periodo de periparto ${ }^{[12,15]}$.

BPC-2.2: Utilizar el instrumento PHQ-9 para el tamizaje de episodio depresivo leve debido a que posee una adecuada sensibilidad (88\%) y especificidad (92\%) ${ }^{[16]}$, fue utilizado en los estudios incluidos, es de uso habitual en la atención primaria de salud; y se encuentra validado en los grupos priorizados en el BPC-2.1 ${ }^{[17-19]}$ y en la población peruana ${ }^{[20,21]}$.

BPC-2.3: Cualquier personal de salud puede realizar el tamizaje en los grupos prioritarios debido a la facilidad del uso del instrumento PHQ-9 y la necesidad de captar un mayor número de pacientes con episodios depresivos.

BPC-2.4: Posterior a un tamizaje positivo se debe confirmar el diagnóstico de episodio depresivo con otros criterios comúnmente utilizados en nuestro contexto, como los basados en el CIE-10 ${ }^{[1]}$, puesto que el tamizaje con PHQ-9 no está exento de clasificar erróneamente a una persona tamizada.

BPC-2.5: Realizar el descarte de depresión bipolar, pues esta condición está presente en aproximadamente $17 \%$ de pacientes con depresión. De no identificar depresión bipolar, la prescripción de monoterapia con antidepresivos puede triplicar el riesgo de síntomas maníacos en estos pacientes ${ }^{[22]}$.

BPC-2.6: La depresión bipolar necesita de un diagnóstico y manejo más especializado, por lo que estos pacientes deben ser referidos al psiquiatra para el diagnóstico confirmatorio y manejo.

BPC-2.7: Se tomó en cuenta los puntos de corte de 10 a 14 del PHQ-19 para definir episodio depresivo leve, en concordancia con lo recomendado por otras GPC ${ }^{[22,23]}$.

BPC-2.8: El manejo de episodios depresivos moderados o severos podrían requerir el uso de terapia combinada, seguimiento más riguroso, y, en algunos casos, manejo hospitalario. Por ello, referir al especialista en psiquiatría en estos casos.

Pregunta 3: En pacientes adultos con episodio depresivo leve, ¿qué terapia se debería brindar como manejo inicial: terapia farmacológica o psicoterapia?

\section{Rec-3.1:}

Para responder esta pregunta se tomó como base la RS de Amick $2015^{[24]}$ que comparó la terapia farmacológica con la psicoterapia en adultos de 18 a 65 años con depresión de al menos severidad moderada. Debido a la antigüedad de la búsqueda en la RS se decidió realizar la actualización de la misma, incluyendo solo el ECA de Schramm $2015^{[25]}$.

Se realizó meta-análisis de los estudios, los cuales finalmente no reportaron diferencias estadísticamente significativas en la remisión de los síntomas depresivos ni en la respuesta al tratamiento (definido como la reducción de los síntomas en por lo menos el $50 \%$ durante el seguimiento).

En cuanto a la factibilidad, muchos de los establecimientos de primer nivel no cuentan con personal capacitado para la realización de psicoterapia, en tanto que otros pueden no contar con terapia farmacológica. Por ello, considerando la similar eficacia encontrada, se decidió favorecer a ambas intervenciones según las necesidades del paciente y el criterio del profesional de salud tratante. Debido a la certeza de la evidencia fue muy baja principalmente debido a que los estudios incluyeron una población de depresión moderada, se decidió emitir una recomendación condicional. 


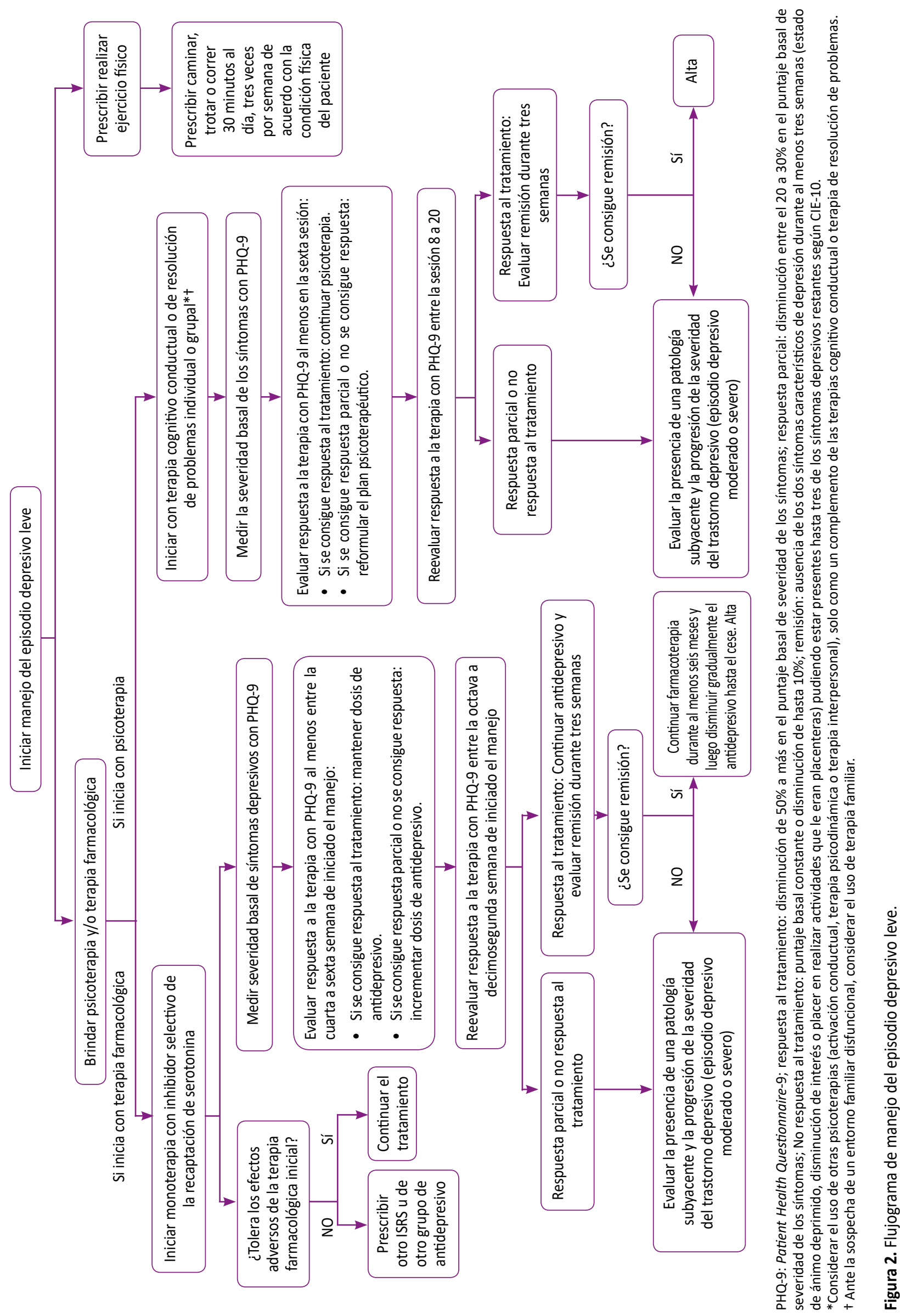


Pregunta 4: En pacientes adultos con episodio depresivo leve en los que se opte por iniciar el manejo con psicoterapia, ¿̇qué psicoterapia se debería usar?

\section{Rec-4.1 y Rec-4.2:}

Para responder esta pregunta se tomó como base la RS de Cuijpers $2014{ }^{[26]}$, la cual se consideró no actualizar debido su autor refirió que no se encontró nuevos estudios relevantes que comparen psicoterapia versus placebo hasta enero de 2018.

La RS de Cuijpers 2014 incluyó 10 ECA con una población de adultos con episodio depresivo de leve a severo. Comparó diversas psicoterapias (TCC, terapia de activación conductual, terapia psicodinámica, terapia interpersonal, y terapia de resolución de problemas) contra placebo para disminuir síntomas depresivos.

Esta RS reportó que la TCC y la terapia de resolución de problemas presentaron mayor reducción de síntomas depresivos en comparación con el grupo placebo (entre estas dos, la TCC tuvo un rendimiento superior). Asimismo, los pocos estudios que evaluaron otras terapias (terapia de activación conductual, terapia psicodinámica y terapia interpersonal) no encontraron diferencias en la reducción de síntomas depresivos al comparar con el placebo.

Debido a que la TCC fue la terapia con mayor rendimiento (moderada certeza de evidencia), se consideró emitir una recomendación fuerte a favor de su uso.

Puesto que la terapia de resolución de problemas fue superior al placebo (muy baja certeza de evidencia), aunque en menor medida que la TCC, se decidió emitir una recomendación condicional a favor de su uso.

BPC-4.1: Si bien no se encontró diferencias estadísticas con el uso de las terapias de activación conductual, psicodinámica y terapia interpersonal, los estudios presentaron limitaciones metodológicas ${ }^{[26]}$. Además, no producirían potenciales daños siempre y cuando sean usadas como complementos. Por ello, podrían brindarse dichas terapias solo para complementar a la TCC o la terapia de resolución de problemas.

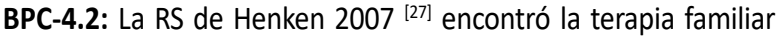
disminuyó la severidad de los síntomas depresivos. Por este motivo, en quienes se sospeche de un entorno familiar disfuncional, se puede usar esta terapia para evitar el reforzamiento de los síntomas depresivos y afianzar las redes de apoyo.

BPC-4.3: Considerar brindar psicoterapia grupal, ya que fue similar de eficaz como la psicoterapia individual, y es importante para fortalecer las redes de apoyo de los pacientes con depresión ${ }^{[26]}$. Además, su implementación implicaría un menor uso de recursos comparado con la psicoterapia individual.

BPC-4.4: La cantidad de sesiones que se brindó en los estudios de la RS de Cuijpers 2014 varió de seis a veinte, una GPC menciona brindar de seis a ocho sesiones ${ }^{[26]}$, y una RS mostró que ofrecer de seis a ocho sesiones de TCC o de terapia de resolución de problemas fue eficaz para reducir los síntomas depresivos ${ }^{[28]}$. Debido a la variabilidad en el número de sesiones, el GEG consensuó que la duración de la psicoterapia sea de 8 hasta 20 sesiones (con una frecuencia de una a dos veces por semana), a criterio del médico tratante considerando la evolución y severidad del cuadro.

BPC-4.5, 4.6, 4.7, 4.8 y 4.9: En pacientes adultos que inician psicoterapia, utilizar el PHQ-9 como instrumento de seguimiento por ser factible de implementar, conocido y validado. Así mismo, se consensó que los periodos de evaluación sean entre la cuarta a octava semana ${ }^{[29]}$, detalles sobre el seguimiento, y las acciones a tomar luego de la evaluación.

Pregunta 5: En pacientes adultos con episodio depresivo leve en los que se opte por iniciar el manejo con terapia farmacológica, ¿con qué antidepresivo se debería iniciar la terapia?

\section{Rec-5.1:}

Para responder esta pregunta se tomó como base la RS y metaanálisis en red de Cipriani $2018^{[30]}$, que incluyó adultos mayores de 18 años con episodio depresivo leve a severo y evaluó la respuesta al tratamiento (reducción mayor o igual a $50 \%$ del puntaje total de una escala de depresión) y aceptabilidad (abandonar el tratamiento) de 21 antidepresivos al compararlos contra placebo y entre sí.

Esta RS encontró que todos los antidepresivos con los que cuenta EsSalud (fluoxetina, sertralina, mirtazapina, venlafaxina, clomipramina y amitriptilina) fueron más eficaces que el placebo para lograr la respuesta al tratamiento. Las diferencias entre los antidepresivos de interés para este desenlace fueron mínimas, por lo cual se consideró que el balance no favorecía significativamente a un grupo farmacológico comparado a los demás.

En cuanto a la aceptabilidad de la terapia, todos a excepción de clomipramina eran tan o más aceptable que placebo. A pesar ser estadísticamente similares en aceptabilidad, los estimados puntuales para ISRS (fluoxetina y sertralina) fueron los mayores, lo cual sugiere que podrían ser más aceptables en comparación con los demás antidepresivos.

El análisis favoreció al uso de antidepresivos frente a placebo y no encontró diferencias significativas respecto al uso de los diversos grupos de antidepresivos. Con respecto a la aceptabilidad, los estimados favorecieron el uso del grupo de antidepresivos ISRS (fluoxetina y sertralina). Considerando que la aceptabilidad es un componente clave del éxito de la terapia ya que mejoraría la adherencia al manejo y podría generar ahorros moderados al evitar un tratamiento prolongado por interrupción o abandono, se optó por emitir una recomendación a favor de iniciar la terapia con ISRS. Dado que no se evaluó efectos adversos, y que su implementación necesitaría de un de un proceso de habilitación para que algunos de los antidepresivos puedan ser prescritos por 
otros especialistas y médicos generales, se decidió emitir una recomendación condicional.

BPC-5.1: Brindar monoterapia como tratamiento dada su eficacia ${ }^{[30]}$. El uso de un segundo antidepresivo en el manejo del episodio depresivo leve podría incrementar la frecuencia de efectos adversos, y por ende de abandono de la terapia. Por otro lado, no iniciar la monoterapia con antidepresivos tricíclicos ya que presentan un mayor riesgo de abandono y pueden generar complicaciones cardiacas ${ }^{[31]}$.

BPC-5.2: En pacientes que presenten efectos adversos con el manejo inicial con ISRS, se puede prescribir otro ISRS o un grupo distinto de antidepresivos, considerando la condición clínica previa del paciente, otros efectos adversos, preferencias del paciente, interacciones con otros medicamentos y la aceptabilidad.

BPC-5.3: La duración de la terapia farmacológica será de 8 hasta 12 semanas ${ }^{[23,29,32]}$. Ampliar dicho periodo dependerá de las necesidades del paciente y del profesional de salud que la realice.

BPC-5.4, 5.5, 5.6 y 5-7: Se estableció por consenso detalles sobre el seguimiento de los pacientes.

Pregunta 6: En pacientes adultos con episodio depresivo leve, ¿se debería indicar realizar ejercicio físico?

\section{Rec-6.1:}

Para responder esta pregunta se tomó como base la RS de Cooney $2013^{[33]}$. Esta incluyó estudios realizados en adultos con depresión principalmente leve a moderada.

Al evaluar efectos a corto plazo (inmediatamente después de terminar las sesiones de ejercicio), con estudios de buena calidad, no se encontró diferencias entre el grupo al que se indicó actividad física en comparación al grupo control. Sin embargo, al evaluar los efectos a largo plazo (entre 4 y 26 meses luego de finalizada la intervención), se encontró menor puntaje de síntomas depresivos en el grupo en el que se indicó ejercicio físico en comparación al control. Al evaluar los dominios de la calidad de vida se encontró diferencias a favor del grupo que recibió ejercicio en el domino ambiental y físico.

Al evaluar los efectos adversos, la RS encuentra siete ECA, pero no realiza meta-análisis debido a la diversidad de eventos reportados, siendo los más frecuentes el dolor muscular, esternal y articular, aunque mencionan que ninguno de los ECA encontró diferencias significativas entre los grupos de ejercicio y control.

Se concluyó que la realización de ejercicios tuvo beneficios en comparación con la no realización, sin haber diferencias en los efectos adversos entre ambos. Por ello, se decidió emitir una recomendación a favor de la realización de ejercicio. Debido a que la certeza de evidencia fue moderada se decidió emitir una recomendación fuerte.
BPC-6.1: En pacientes con episodio depresivo leve puede prescribirse ejercicios tanto aeróbicos como de resistencia ${ }^{[3]}$. Asimismo, elegir una frecuencia de ejercicios de tres veces por semana por 30 minutos debido a que esta fue prescrita en la mayoría de ECA incluidos en la RS evaluada.

BPC-6.2: La actividad física se prescribirá como un complemento de la terapia farmacológica o psicoterapia y no las reemplazará.

Agradecimientos: Agradecemos al Dr. Rubén Valle Rivadeneyra, médico psiquiatra del Instituto Nacional de Salud Mental "Honorio Delgado - Hideyo Noguchi"; y al Dr. Juan Antonio Gálvez-Buccollini Abanto, médico psiquiatra de la Clínica Anglo Americana; por la revisión de la presente guía de práctica clínica.

Contribuciones de autoría: Todos los autores participaron en la realización de la guía. JHZT y SGL se encargaron de las búsquedas sistemáticas, la evaluación de calidad de los estudios y la evaluación de la certeza de la evidencia para cada pregunta. RNMR y WNG redactaron la primera versión del artículo. Todos los autores participaron en la discusión de los estudios encontrados y la formulación de las recomendaciones y puntos de buenas prácticas clínicas. Todos los autores revisaron y aprobaron la versión final del artículo.

Potenciales conflictos de intereses: Los responsables de la elaboración del presente documento declaran no tener ningún conflicto de interés financiero o no financiero, con relación a los temas descritos en el presente documento.

Fuentes de financiamiento: Este documento técnico ha sido financiado por el Instituto de Evaluación de Tecnologías en Salud e Investigación (IETSI), EsSalud, de Perú.

\section{ORCID}

Beatrice Macciotta Felices, https://orcid.org/0000-0002-10051106

Carla Morón Corales, https://orcid.org/0000-0001-8990-8530

Matilde Luna Matos, https://orcid.org/0000-0002-7789-8619

Víctor Gonzales Madrid, https://orcid.org/0000-0001-5888-9189

Amelia Melgarejo Moreno, https://orcid.org/0000-0002-39306256

Jessica Hanae Zafra-Tanaka, https://orcid.org/0000-0001-63866643

Sergio Goicochea-Lugo, https://orcid.org/0000-0002-0487-5547 Raisa N. Martinez-Rivera, https://orcid.org/0000-0002-36117628

Wendy Nieto-Gutierrez, https://orcid.org/0000-0001-8012-1520 Fabián Fiestas-Saldarriaga, https://orcid.org/0000-0002-9158443X

Alvaro Taype-Rondan, https://orcid.org/0000-0001-8758-0463

Raúl Timaná Ruiz, https://orcid.org/0000-0003-0693-3445

Hector Garavito Farro, https://orcid.org/0000-0002-9590-9027 


\section{REFERENCIAS BIBLIOGRÁFICAS}

1. World Health Organization. ICD-10, international statistical classification of diseases and related Health problems [Internet]. Geneva, Switzerland: WHO Press; 2010 [citado el 23 de abril de 2020]. Disponible en: https://www.who.int/classifications/icd/en

2. American Psychiatric Association. Diagnostic and statistical manual of mental disorders (DSM). 5th ed. USA: American Psychiatric Association; 2013.

3. World Health Organization. Fact sheets: Depression [Internet]. Suiza: WHO; 2019 [citado el 23 de abril de 2020]. Disponible en: https:// www.who.int/news-room/fact-sheets/detail/depression

4. Timonen $\mathrm{M}$, Liukkonen $\mathrm{T}$. Management of depression in adults. BMJ. 2008;336(7641):435-9. doi: 10.1136/bmj.39478.609097.BE.

5. Gautam S, Jain A, Gautam M, Vahia VN, Grover S. Clinical Practice Guidelines for the management of Depression. Indian J Psychiatry. 2017;59(Suppl 1):S34-S50. doi: 10.4103/0019-5545.196973.

6. Villarreal-Zegarra D, Cabrera-Alva M, Carrillo-Larco RM, BernabeOrtiz A. Trends in the prevalence and treatment of depressive symptoms in Peru: a population-based study. BMJ Open. 2020;10(7):e036777. doi: 10.1136/bmjopen-2020-036777.

7. Toyama M, Castillo H, Galea JT, Brandt LR, Mendoza M, Herrera V, et al. Peruvian Mental Health Reform: A Framework for Scaling-up Mental Health Services. Int J Health Policy Manag. 2017;6(9):501-8. doi: 10.15171/ijhpm.2017.07.

8. Balshem $\mathrm{H}$, Helfand $\mathrm{M}$, Schünemann HJ, Oxman AD, Kunz R, Brozek J, et al. GRADE guidelines: 3 . Rating the quality of evidence. J Clin Epidemiol. 2011;64(4):401-6. doi: 10.1016/j.jclinepi.2010.07.015.

9. Andrews J, Guyatt G, Oxman AD, Alderson P, Dahm P, Falck-Ytter Y, et al. GRADE guidelines: 14. Going from evidence to recommendations: the significance and presentation of recommendations. J Clin Epidemiol. 2013;66(7):719-25. doi: 10.1016/j.jclinepi.2012.03.013.

10. Conejo-Cerón S, Moreno-Peral P, Rodriguez-Morejon A, Motrico E, Navas-Campana D, Rigabert A, et al. Effectiveness of psychological and educational interventions to prevent depression in primary care: a systematic review and meta-analysis. Ann Fam Med. 2017;15(3):262-71. doi: 10.1370/afm.2031.

11. Driessen E, Hollon SD. Cognitive behavioral therapy for mood disorders: efficacy, moderators and mediators. Psychiatr Clin North Am. 2010;33(3):537-55. doi: 10.1016/j.psc.2010.04.005.

12. Siu AL, Bibbins-Domingo K, Grossman DC, Baumann LC, Davidson $\mathrm{KW}$, Ebell $\mathrm{M}$, et al. Screening for depression in adults: US Preventive Services Task Force recommendation statement. JAMA. 2016;315(4):380-7. doi: 10.1001/jama.2015.18392.

13. Williams Jr JW, Mulrow CD, Kroenke K, Dhanda R, Badgett RG, Omori $\mathrm{D}$, et al. Case-finding for depression in primary care: a randomized trial. Am J Med. 1999;106(1):36-43. doi: 10.1016/ s0002-9343(98)00371-4.

14. Silverstone PH, Rittenbach K, Suen VY, Moretzsohn A, Cribben I, Bercov $M$, et al. Depression outcomes in adults attending family practice were not improved by screening, stepped-care, or online CBT during a 12-week study when compared to controls in a randomized trial. Front Psychiatry. 2017;8:32. doi: 10.3389/ fpsyt.2017.00032.

15. Martina $M$, Ara MA, Gutiérrez $C$, Nolberto V, Piscoya J. Depresión y factores asociados en la población peruana adulta mayor según la ENDES 2014-2015. An Fac med. 2017;78:393-7. doi: 10.15381/ anales.v78i4.14259.

16. Baader M T, Molina F JL, Venezian B S, Rojas C C, Farías S R, FierroFreixenet C, et al. Validación y utilidad de la encuesta PHQ-9 (Patient Health Questionnaire) en el diagnóstico de depresión en pacientes usuarios de atención primaria en Chile. Rev Chil Neuro-Psiquiat. 2012;50:10-22. doi: 10.4067/S0717-92272012000100002

17. Phelan E, Williams B, Meeker K, Bonn K, Frederick J, Logerfo J, et al. A study of the diagnostic accuracy of the PHQ-9 in primary care elderly. BMC Fam Pract. 2010;11:63. doi: 10.1186/1471-2296-11-63.

18. Gjerdingen D, Crow S, McGovern P, Miner M, Center B. Postpartum depression screening at well-child visits: validity of a 2-question screen and the PHQ-9. Ann Fam Med. 2009;7(1):63-70. doi: 10.1370/afm.933.

19. Richardson LP, McCauley E, Grossman DC, McCarty CA, Richards J Russo JE, et al. Evaluation of the Patient Health Questionnaire- 9 Item for detecting major depression among adolescents. Pediatrics. 2010;126(6):1117-23. doi: 10.1542/peds.2010-0852.

20. Calderón M, Gálvez-Buccollini JA, Cueva G, Ordoñez C, Bromley C, Fiestas F. Validación de la versión peruana del PHQ-9 para el diagnóstico de depresión. Rev Peru Med Exp Salud Publica. 2012;29:578-9.

21. Villarreal-Zegarra D, Copez-Lonzoy A, Bernabé-Ortiz A, MelendezTorres GJ, Bazo-Alvarez JC. Valid group comparisons can be made with the Patient Health Questionnaire (PHQ-9): A measurement invariance study across groups by demographic characteristics. PLoS One. 2019;14(9):e0221717. doi: 10.1371/journal.pone.0221717.

22. Grupo de trabajo de la Guía de Práctica Clínica sobre el Manejo de la Depresión en el Adulto. Guía de Práctica Clínica sobre el Manejo de la Depresión en el Adulto. Ministerio de Sanidad, Servicios Sociales e Igualdad [Internet]. España: Agencia de Evaluación de Tecnologías Sanitarias de Galicia (avalia-t); 2014 [citado el 23 de abril de 2020]. Disponible en: https://semap.org/docs/GPC.pdf

23. Management of Major Depressive Disorder Working Group. VA/ DoD clinical practice guideline for the management of major depressive disorder [Internet]. USA: Department of Veterans Affairs; 2016 [citado el 23 de abril de 2020]. Disponible en: https://www. healthquality.va.gov/guidelines/MH/mdd/

24. Amick HR, Gartlehner G, Gaynes BN, Forneris C, Asher GN, Morgan $L C$, et al. Comparative benefits and harms of second generation antidepressants and cognitive behavioral therapies in initial treatment of major depressive disorder: systematic review and meta-analysis. BMJ. 2015;351:h6019. doi: 10.1136/bmj.h6019.

25. Schramm E, Zobel I, Schoepf D, Fangmeier T, Schnell K, Walter H, et al. Cognitive Behavioral Analysis System of Psychotherapy versus Escitalopram in Chronic Major Depression. Psychother Psychosom. 2015;84(4):227-40. doi: 10.1159/000381957.

26. Cuijpers P, Turner E, Mohr DC, Hofmann S, Andersson G, Berking $M$, et al. Comparison of psychotherapies for adult depression to pill placebo control groups: a meta-analysis. Psychol Med. 2014;44(4):685-95. doi: 10.1017/S0033291713000457.

27. Henken HT, Huibers MJ, Churchill R, Restifo K, Roelofs J. Family therapy for depression. Cochrane Database Syst Rev. 2007;2007(3):Cd006728. doi: 10.1002/14651858.cd006728.

28. Nieuwsma JA, Trivedi RB, McDuffie J, Kronish I, Benjamin D, Williams JW. Brief psychotherapy for depression: a systematic review and meta-analysis. Int J Psychiatry Med. 2012;43(2):129-51. doi: 10.2190/PM.43.2.C.

29. American Psychiatric Association. Practice guideline for the treatment of patients with major depressive disorder [Internet]. USA: American Psychiatric Association; 2010 [citado el 23 de abril de 2020]. Disponible en: https://psychiatryonline.org/pb/assets/ raw/sitewide/practice guidelines/guidelines/mdd.pdf.

30. Cipriani A, Furukawa TA, Salanti G, Chaimani A, Atkinson LZ, Ogawa $Y$, et al. Comparative efficacy and acceptability of 21 antidepressant drugs for the acute treatment of adults with major depressive disorder: a systematic review and network meta-analysis. Focus (Am Psychiatr Publ). 2018;16(4):420-9. doi: 10.1176/appi. focus.16407.

31. Coupland C, Hill T, Morriss R, Moore M, Arthur A, Hippisley-Cox J. Antidepressant use and risk of cardiovascular outcomes in people aged 20 to 64: cohort study using primary care database. BMJ. 2016;352:i1350. doi: 10.1136/bmj.i1350.

32. Cleare A, Pariante CM, Young AH, Anderson IM, Christmas D, Cowen PJ, et al. Evidence-based guidelines for treating depressive disorders with antidepressants: A revision of the 2008 British Association for Psychopharmacology guidelines. J Psychopharmacol. 2015;29(5):459-525. doi: 10.1177/0269881115581093.

33. Cooney GM, Dwan K, Greig CA, Lawlor DA, Rimer J, Waugh FR, et al. Exercise for depression. Cochrane Database Syst Rev. 2013(9):Cd004366. doi: 10.1002/14651858.CD004366.pub6. 